\title{
BILATERAL OPTIC ATROPHY IN CHILDHOOD. II
}

\author{
Optic Atrophy as the Predominant Clinical Feature
}

\section{(1) Following Meningitis or Encephalomyelitis}

Optic neuritis may occasionally occur as a complication of infective meningitis or as one aspect of the widespread encephalomyelitis sometimes complicating exanthematous illnesses, while a true papilloedema may also occur in these conditions. In some cases the most significant permanent neurological deficit after recovery from the initial illness is defective vision due to bilateral optic atrophy; this is occasionally due to the toxic effects of sulphonamide, isoniazid or streptomycin therapy.

\section{(2) Following Head Injury}

Head injury may occur either at birth or later in childhood and may cause optic atrophy in a number of ways: a fracture line may involve both optic foramina; intraneural haemorrhage may be caused by a contrecoup injury; or a subdural effusion may accumulate giving rise to optic atrophy either by stretching of the optic nerves, or as a result of papilloedema.

\section{(3) Following Lead Poisoning}

Lead poisoning in children is usually acute after the ingestion of lead-containing paint. It produces a severe encephalopathy which may lead to cortical blindness. In some, however, a permanent visual defect is caused by optic atrophy resulting from toxic optic neuritis or papilloedema.

\section{(1) Hydrocephalus}

\section{Optic Atrophy as One Aspect of a Generalized Disorder}

This may be due either to a developmental abnormality or to the consequences of meningitis, trauma, or neoplasia, and is a common cause of optic atrophy in early childhood. The atrophic changes may be due to the stretching and angulation of the optic nerves which occur as the skull expands, or they may be secondary to papilloedema. Papilloedema is rare in untreated cases, except in hydrocephalus due to a thrombosis of the superior longitudinal sinus, but where the intracranial pressure has been reduced by the introduction of a ventriculo-atrial shunt, premature synostosis of the skull may occur. If the shunt subsequently becomes blocked there is commonly a rapid rise in the intracranial pressure with the appearance of papilloedema; if this complication is not quickly alleviated it may lead to optic atrophy.

\section{(2) Cerebral Palsy}

This may result from one of a number of processes which affect the foetus or the newborn baby, the most common being neonatal asphyxia. In many cases, however, there is no apparent reason for the arrest of cerebral differentiation which occurs late in foetal development. In severely affected children there is a high incidence of optic atrophy which represents just one aspect of the global cerebral deficit.

\section{(3) Microcephaly}

A severe deficiency in cerebral development causes microcephaly or porencephaly and in many cases this lack of cerebral differentiation, which results in amentia, is associated with optic atrophy.

\section{(4) Lipidoses and Leucodystrophies}

In these rare hereditarily-determined disorders optic atrophy is a very common finding and in some instances an acquired visual defect is the presenting sign.

THE LIPIDOSES are characterized by the accumulation of abnormal lipids in various body tissues. All cases run a progressive and fatal course. The predominant clinical feature is the loss of previously acquired motor skills and intellectual deterioration. 
(a) Ganglioside lipidoses. (i) Congenital form. This is very rare and optic atrophy is associated with macular pigmentation. (ii) Infantile form (Tay-Sachs). The onset of symptoms usually occurs at 6 months of age, with irritability and delayed development followed by progressive weakness, spasticity, convulsions, and dementia. An exaggerated startle response to sound is a constant and very early feature. Death and swelling of the retinal ganglion cells causes the cherryred spot and consecutive optic atrophy; with this progressive loss of vision a pendular nystagmus develops. A deficiency of the enzyme fructose-1-phosphate aldolase has been noted both in affected patients and in heterozygous relatives. Death occurs before the age of 3 years. (iii) Late infantile form (Bielschowsky-Jansky). This ill-defined entity has its onset at about 3 years of age and lasts up to 6 years. A cherry-red spot or pigmentation at the macula may be associated with optic atrophy. (iv) Juvenile form (Vogt-Spielmeyer; Batten-Mayou). This develops between 5 and 8 years of age and lasts as long as 10 to 15 years. Tremors, muscular rigidity, and athetosis are common features and blindness may occur early. A widespread tapeto-retinal degeneration results in pigmentation of the macula as well as of the peripheral retina. (v) Adult form (Kufs). This presents as a disorder of the cerebellum or basal ganglia, the eyes being unaffected.

(b) Sphingomyelin lipidosis (Niemann-Pick). - In the acute infantile form, in which there is an accumulation of sphingomyelin in reticulum cells, physical and mental retardation are associated with cranial nerve palsies, wasting of the extremities and hepatosplenomegaly. A cherry-red spot at the macula occurs in about half the cases and a waxy atrophy of the optic discs is commonly seen.

THE LEUCODYSTROPHIES are an incompletely understood group of disorders occurring most commonly in infancy and early childhood and characterized by a diffuse and progressive degeneration of the white matter of the central nervous system without inflammatory reaction. Metachromatic leucodystrophy (sulphatide lipidosis) is as yet the only member of the group in which the specific error of metabolism is at all understood. Sulphatides can be demonstrated by metachromatic staining reactions in the white matter, myelin sheaths, renal tubules, and urinary deposits. Optic atrophy is an early and progressive feature of all three forms of this disorder. (a) Infantile form. Develops between 1 and 2 years with ataxia and an inability to walk and talk, followed by visual deterioration leading to blindness and progressive dementia. (b) Juvenile form. Develops between 6 and 10 years of age. Cerebellar signs, deafness and blindness (due to optic atrophy) are followed by spasticity, fits, and dementia. (c) Adult form. Usually develops after puberty and presents with visual disturbances, ataxia, and dysarthria, followed by quadriplegia.

Other forms of leucodystrophy are distinguishable on clinical and histological grounds. All exhibit progressive optic atrophy, but the most important to the ophthalmologist is the sudanophilic type, comprising most cases of Schilder's disease which usually present as cortical blindness.

\section{(5) Hereditary Ataxias}

Behr's optic atrophy, a congenital form of hereditary ataxia, is inherited as an autosomal recessive trait and is characterized by severe bilateral optic atrophy, pyramidal signs, ataxia, club-foot, and mental subnormality. Nystagmus is present in about half the cases and strabismus in two-thirds. The ERG is normal. Optic atrophy is occasionally seen in Friedreich's ataxia in children.

\section{(6) Craniostenoses}

In this group there is a premature fusion of one or more of the cranial sutures leading to deformity in the growth of the skull. Atrophic changes in the optic nerves are very common, resulting from stretching and angulation of the intracranial portions of the optic nerves and of the optic chiasma, papilloedema or, rarely, from compression of the optic nerves in the optic canals.

\section{(7) Congenital Syphilis}

Bilateral optic atrophy following optic neuritis is a rare manifestation of this disorder; more commonly atrophic changes in the optic nerves are secondary to a diffuse chorio-retinitis or to a basal syphilitic meningitis, or are associated with congenital tabes or general paralysis.

Brian Harcourt ANd Barrie Jay, INSTITUTE OF OPHTHALMOLOGY, JudD ST., LoNDON, W.C.1. 